\title{
REVIEWING ARTICLE: ISCHAEMIC AND METABOLIC TREATMENT OF HEPATIC TUMOURS
}

\author{
BO AHRÉN* and STIG BENGMARK \\ Department of Surgery, Lund University, Lund, Sweden
}

\begin{abstract}
For treatment of malignancies, physical and metabolic differences between tumour cells and host cells have guided the development of new approaches. In this review, two new approaches to be used in the treatment of liver malignancies are outlined: ischaemic therapy and interferences with the glucose metabolism. Ischaemic therapy of liver malignancies has been used in different forms during the last 20 years: from ligation of the hepatic artery, embolization of the arterial tree, transient occlusion of the hepatic artery to the present day use of temporary, intermittent, transient hepatic arterial occlusion. The beneficial effect of ischaemic therapy on malignancies is supposed to depend on oxygen and nutritional deficiency, formation of oxygen-derived free radicals and loss of function in cellular enzymes. The tumour cells seem thereby to be more sensitive than the host cells. Also, ischaemia might potentiate the effect of cytotoxic drugs. Intereferencies with glucose metabolism might be directed either towards the exaggerated tumour glycolysis, for example by glucose analogues like 2-deoxy-glucose, or towards the exaggerated host gluconeogenesis, for example by hydrazine sulphate. These treatments result in reduction of the glucose availability in the intracellular glucose metabolism in the tumour cells and have experimentally been demonstrated to be correlated to reduced tumour growth. It is concluded that both these approaches, ischaemic therapy and manipulations with the glucose metabolism, seem promising for the future. What is needed now is research to clarify the mechanims behind the effects, to establish their full consequences, and to identify the clinical use of these treatments and their possible combinations.
\end{abstract}

KEY WORDS: Cancer, malignancy, liver, ischaemia, treatment, glucose metabolism, glucose oxidation, glycolysis, 2-deoxy-glucose, gluconeogenesis, hydrazine.

\section{INTRODUCTION}

For treatment of malignancies, physical and metabolic differences between tumour cells and host cells have guided the development of new approaches. For example, the greater sensitivity to cytotoxic agents in rapidly growing tumour cells compared to that in normal cells has made feasible the intense use of various cytotoxic drugs for treatment. Likewise, a sensitivity for radiation and hyperthermia greater in tumour cells than in normal cells has firmed the use of these models in cancer treatment. We will here focus attention on two other new approaches to be used in the treatment of malignancies: ischaemic therapy and interferences with the tumour glucose metabolism. For both these new approaches, differences in tumour versus host-cell function may explain the beneficial effects, and, furthermore, knowledge of these differences may be used when optimizing the therapy.

\footnotetext{
* Correspondence and reprint requests to: Dr Bo Ahrén, Department of Surgery, Lund University, 22185 Lund, Sweden.
} 


\section{HEPATIC DEARTERIALIZATION}

Ischaemic therapy of liver malignancy was intitiated when it was realized that liver tumour cells preferentially are nourished by the arterial blood supply, whereas hepatocytes, on the contrary, are supplied mainly by portal blood ${ }^{1,2}$. Exceptions exist, however, since cells in small tumours and cells located peripherally in the tumours seem to be nourished also by the portal blood ${ }^{3,4}$. In the earlier phase of this development, ligation or embolization of the hepatic artery was performed ${ }^{5-8}$. This manipulation was found to reduce tumour growth, at least initially, but was also found to initiate collateral formation, which counteracts the aim of the ligation ${ }^{9}$. Therefore, a method of temporary dearterialization was developed: slings were introduced around the hepatic artery and removed after 16 hours ${ }^{10}$. This temporary dearterialization seemed to prevent collateral formation, but the technique was a one-shot treatment, which to be repeated necessitated a new laparatomy. Nevertheless, it was found to be beneficial for carcinoid tumours, with regard both to tumour volume reduction and to improvement of the carcinoid syndrome ${ }^{11}$.

Recently, a new device allowing repeated hepatic arterial occlusion has been developed $^{12}$. The occluder is connected to a subcutaneous injection port through which fluid may be injected, leading to hepatic artery occlusion (Figures 1 and 2). It is hoped that the repeatedly undertaken temporary dearterialization may prevent collateral formation and still retain the tumour reduction effect ${ }^{13}$. It has to be emphasized here that these arterial occlusion models all need the performance of an extensive devascularization procedure of the liver to make the hepatic artery the only supplier of arterial blood to the liver.

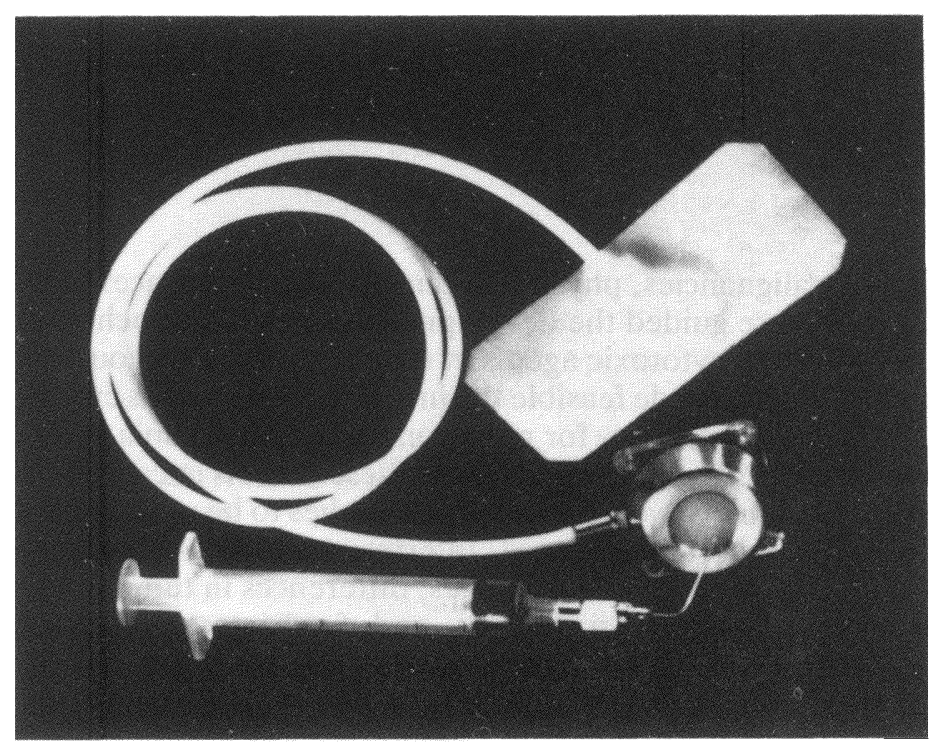

Figure 1 Implantable device for intermittent, repeatedly undertaken dearterialization of the liver. 


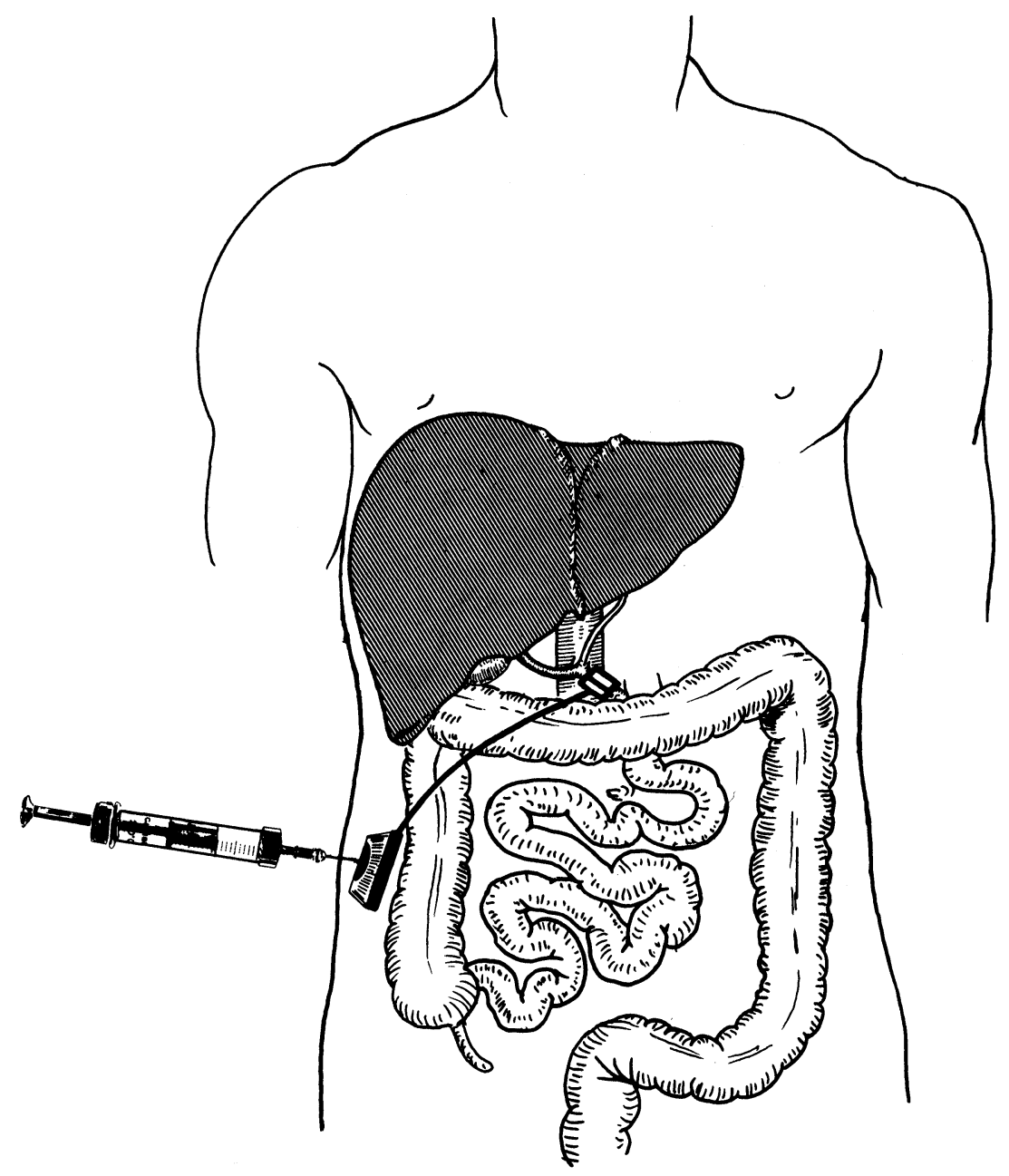

Figure 2 The implantable occluder applied to the hepatic artery and connected to a port.

\section{Ischaemic Effects of Dearterialization}

A main effect of hepatic dearterialization is the resulting ischaemia. This eventually causes hypoxia in the tumours, as has been demonstrated by the clamping of experimental rodent tumours ${ }^{14}$. The oxygen lack halts cellular respiration, which subsequently results in cell damage. That oxygen lack indeed results in tumour necrosis has been demonstrated in a multicellular spheroid model of tumour microregions ${ }^{15}$. However, the same study also showed that necrosis may develop despite adequate oxygen supply, illustrating that other factors, such as glucose deprivation, acidosis or lactate accumulation, may also be of importance for necrosis development ${ }^{15}$. It is of interest to mention that small tumours might be more sensitive to ischaemia than large tumours, since they have a higher oxygen 
consumption rate ${ }^{16}$. Although tumour cells might thus be injured by the ischaemia occuring during dearterialization, the oxygen lack might also influence normal liver cells. A goal in this mode of treatment is to minimize the negative influence on the normal cells and still retain the beneficial effects on the tumours. Before reaching this goal, we have to analyze the characteristics and extent of ischaemia-induced injuries to the normal liver. It must, however, be mentioned that the tumours seem to be more sensitive to hypoxia than normal cells, probably because the rapid proliferation, notably of the tumour vascular endothelium ${ }^{17}$.

Injured normal liver cells during and immediately after ischaemia have been demonstrated by the use of histochemical dye techniques ${ }^{18}$. Flocculent densities in the mitochondrial areas, as a sign of cell injury, have also been shown at $24 \mathrm{~h}$ after liver ischaemia in the rat ${ }^{19}$. Furthermore, the activity of the mitochondrial enzyme glutamate dehydrogenase is concomitantly decreased ${ }^{19}$. Moreover, after $3 \mathrm{~h}$ ischaemia of the rat liver, the activity of phospholipase $\mathrm{A}$ in the mitochondria has increased, which has resulted in accumulation of lysophospholipids and a subsequent injured control of the innner mitochondrial layer ${ }^{20}$. Also, after $5 \mathrm{~h}$ ischaemia of the rat liver, serum levels of the mitochondrial enzyme ornithine carbamyl transferase have increased ${ }^{21}$. Injuries to the mitochondria seem, therefore, to be a common manifestation of liver ischaemia. However, other hepatic functions might also be altered by ischaemic treatment. Thus, microvilli of the bile canaliculi have been demonstrated to disappear after $15 \mathrm{~min}$ ischaemia, and the activity of the plasma membrane enzyme 5'-mononucleotidase is lowered after $30 \mathrm{~min}$ ischaemia ${ }^{22}$. Lysosomal alterations have also been seen during hepatic ischaemia: the serum activity of the lysosomal enzyme $\beta$-glucosaminidase in the rat is lowered after $1 \mathrm{~h}$ hepatic ischaemia ${ }^{23}$, and serum lysosomal enzyme activities in man are increased after hepatic dearterialization ${ }^{24}$. In addition, hypoxia and ischaemia in the perfused pig liver have been shown to impair the hepatic galactose elimination rate, inhibit ATP phosphorylation and increase the lactate output ${ }^{25}$. However, depite $80 \mathrm{~min}$ ischaemia, almost complete recovery occurred ${ }^{25}$.

\section{Other Effects of Dearterialization}

Besides the hypoxic-ischaemic effects of clamping procedures of the hepatic artery, these also seem to increase sensitivity to radiation and cytotoxic drugs, induce the formation of oxygen-derived free radicals, and restrict the nutritional supply to the tumours. These effects might also be of importance, perhaps as important as the ischaemic-hypoxic effects of dearterialization. That clamping enhances sensitivity to radiation has been demonstrated in rodent mammary tumours ${ }^{14}$, and the potentiation of cytostatic drugs is exemplified by the anticancer agent mitomycin $\mathrm{C}$, which has been demonstrated to be more cytotoxic to hypoxic tumour cells than to cells under normal oxygen tension ${ }^{26}$. Likewise, cisplatin seems to reduce the oxidative metabolism in polymorphonuclear cells, which might be indicative of a hypoxia-like action, which then will be further enhanced by hypoxia ${ }^{27}$.

Ischaemic therapy has also been demonstrated to induce the formation of oxygenderived free radicals ${ }^{28}$. These toxic substances (superoxide, hydrogen peroxide and hydroxyl radicals) are produced under hypoxic conditions and during reperfusion after hypoxia ${ }^{29}$. A mechanism behind their production is the reduction of hypoxanthine to xanthine by the enzyme xanthine dehydrogenase, which under hypoxic conditions functions as a xanthine oxidase ${ }^{30}$. Under normal conditions, 
protective mechanisms against this conversion exist, but these are impaired during hypoxia $^{31}$. Consequently, xanthine oxidase inhibition by allopurinol has been demonstrated to prevent a long-standing reduction in protein synthesis after liver ischaemia in rats ${ }^{32}$, and the radical scavenger disulfiram has been demonstrated to reduce the number of injured liver cells after $90 \mathrm{~min}$ ischaemia in rats ${ }^{33}$. Likewise, in one study, the scavenger glutathione was protective against rat liver ischaemia when intracellular calcium distribution, dye exclusion and membrane potential were investigated ${ }^{34}$. Hence, dearterialization might be deleterious to cells also through the production of oxygen-derived free radicals ${ }^{35}$.

Clamping of the arterial supply reduces the nutritional supply to the cells. To what extent this may explain the cell injury and whether tumour cells are more vulnerable to this effect are questions that remain to be settled. However, it has been shown that protein synthesis in the liver is reduced by ischaemia ${ }^{36-37}$, and it has also been demonstrated that glucose deprivation to tumour cells in vitro results in derangements of protein synthesis similar to those from hypoxia ${ }^{38}$. Furthermore, central tumour necrosis is induced not only by hypoxia but also by lack of glucose ${ }^{15}$.

Thus, effects other than pure hypoxia seem to evolve by clamping and dearterialization. Of these, exaggerated sensitivity to cytotoxic treatment, superoxide production and deprivation of the nutritional supply seem to be most interesting in relation to tumour treatment. Combined treatment might be scheduled upon this knowledge.

\section{Dearterialization: Conclusions}

As outlined in the discussion so far, the full consequences and exact mechanism of ischaemic therapy of liver malignancy are not established. It is clear, however, that not only hypoxic effects are induced, but also exaggerated sensitivities to cytotoxic substances, formation of oxygen-derived free radicals, and reduction of the nutritional supply. Experimentally, ischaemia has been shown to impair tumour growth $^{39}$ and, for future development and research, a small implantable device for repeated temporary hepatic dearterialization in the rat has been developed ${ }^{40}$. It will be used to study the consequences of hepatic ischaemia and to optimize treatment. Clinically, hepatic dearterialization used for $16 \mathrm{~h}$ has been shown to reduce carcinoid tumour growth ${ }^{11}$. Studies on the theoretically more optimal repeated temporary dearterialization are under way. Future research must establish the time dependency of effects on the tumour and the host cells to settle the optimal schedule for treatment. The optimal treatment should use the beneficial effect of tumour growth retardation but avoid ischaemic effects on normal liver cells. At present, a schedule of hepatic dearterialization $1 \mathrm{~h}$ twice daily is considered most proper and used in our department.

\section{MANIPULATION OF GLUCOSE METABOLISM}

Several different types of tumour cells are characterized by deriving their energy mainly from the glycolytic pathway and thus they show a great dependency on glucose supply for their survival. This characteristic was recognized several decades $\mathrm{ago}^{41}$ and has recently been reconfirmed by the demonstration that tumours exhibit 
high rates of glucose utilization and lactic acid production ${ }^{42}$. This high rate of glycolysis by tumours could be a mechanism behind the exaggerated glucose turnover rate in tumour patients, as shown by the glucose isotope tracer technique ${ }^{43,44}$. This abnormally high dependency on glucose is a potential difference between tumour and host cells to which intervention may be directed. Two different approaches may then be undertaken: reduction of the glycolytic activity and restrainment of the glucose supply.

\section{Inhibition of Glycolysis}

The glycolytic pathway is the process in which glucose is converted to substrates for the tricarboxylic acid cycle and in which the energy of the glucose molecule is liberated as ATP. The pathway involves several different enzyme steps converting glucose-6-phosphate to pyruvic and lactic acid (Figure 3). The glycolytic flow can be inhibited by the substance 2-deoxyglucose, which is a glucose analogue (Figure 4). 2Deoxyglucose inhibits both the glucose uptake into the cell, and the hexokinase that catalyzes the phosphorylation of glucose (i.e. the formation of glucose-6phosphate $)^{45,46}$. 2-Deoxyglucose is by itself converted to a 2-deoxyglucose-6phosphate, but this substance is not isomerized to fructose-6-phosphate, and thus does not enter the glycolytic pathway further. 2-Deoxyglucose might also inhibit the conversion of glucose-6-phosphate to glucose-1-phosphate ${ }^{47}$. A marked reduction in glycolysis by 2 -deoxyglucose has been demonstrated in vitro in leukaemic leukocytes and in HeLa cells ${ }^{48,49}$. Since tumour cells, as outlined above, seem more dependent on this pathway for their energy supply than other cells, 2-deoxyglucose might be used in the treatment of malignancies. This has been investigated experimentally in a few studies. In one study, the effects of 2-deoxyglucose on tumour growth in three different tumour models in the mouse were investigated: in a leukaemia, a transplantable carcinoma, and a mast cell tumour ${ }^{50}$. 2-Deoxyglucose was found to reduce tumour growth and induce a prolongation of the survival time $\mathrm{e}^{50}$. Furthermore, a recent study on fibrosarcoma in the rat demonstrated that upon systemic administration 2-deoxyglucose reduced the tumour growth ${ }^{51}$, and a recent

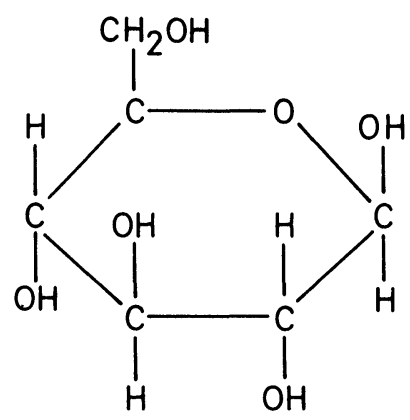

Glucose

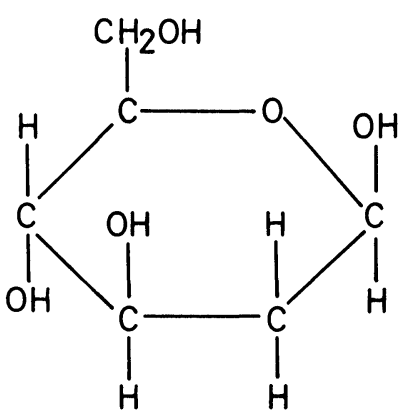

2-Deoxy-glucose

Figure 4 The chemical structures of glucose and 2-deoxyglucose, illustrating that 2-deoxyglucose lacks the hydroxyl group in carbon position 2 . 

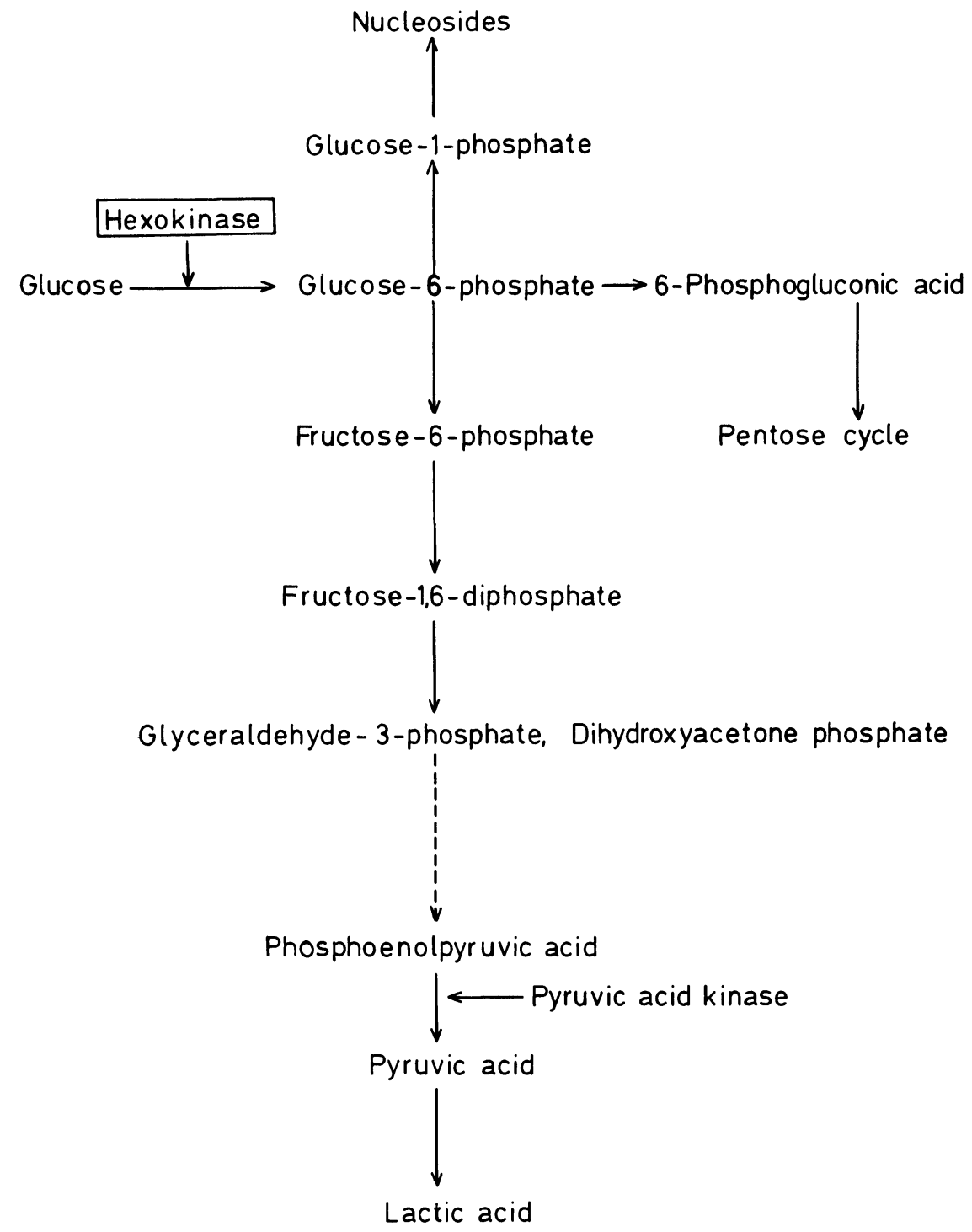

Figure 3 A simplified outline of the intracellular metabolic fate of glucose, showing the glycolytic pathway in which glucose-6-phosphate is converted to pyruvic and lactic acid. 2-Deoxyglucose inhibits this pathway by inhibiting the cellular uptake of glucose and by inhibiting the enzyme hexokinase. Also illustrated in the figure is the enzyme pyruvic acid kinase, which in glycolysis converts phosphoenolpyruvic acid to pyruvic acid.

study on HeLa cells showed that 2-deoxyglucose at high-dose levels inhibited DNA repair after radiation ${ }^{49}$. However, in two other experimental tumour models in the mouse, a fibrosarcoma and a mammary adenocarcinoma, 2-deoxyglucose failed to alter tumour growth when administered at dose levels high enough to produce systemic toxicity ${ }^{52}$. This illustrates that different tumours might respond with 
different sensitivity to the inhibition of glycolysis, and that the systemic toxicity of glucose analogues might restrict their use. The toxicity depends on the neuroglycopenia, since a competition with glucose in the glycolytic pathway also occurs within the brain, which is strictly dependent on glucose for its metabolism. Neuroglycopenia causes neurological disturbances that may lead to seizures and death $^{52}$. Also, neuroglycopenia induces reflex activation of the autonomic system, as evidenced by enhanced plasma levels of noradrenaline ${ }^{53}$ and a cholinergically mediated stimulation of insulin secretion ${ }^{54}$.

In conclusion, the approach of inhibiting the glycolytic pathway in tumours seems valid and might guide future research. What is needed is, first, to establish which malignancies are sensitive to this mode of treatment and, secondly, to develop inhibitors of glycolysis that exert effects on tumour metabolism without affecting neural glycolysis. Regional administration of glucose analogues might also reduce toxic systemic effects. Also, since hypoxic cells seem more influenced by glucose deprivation than normal cells, the combined use of glucose analogues and dearterialization procedures has to be investigated.

\section{Inhibition of Gluconeogenesis}

The formation of glucose from non-carbohydrate precursors, mainly amino acids and lactate, is called gluconeogenesis (Figure 5). This pathway is often exaggerated in cancer patients because of the general catabolism, with an exaggerated production of amino acids from the protein breakdown, of glycerol from the massive lipid mobilization, and of lactate from the enhanced glycolytic pathway in the tumour ${ }^{55}$. This massive gluconeogenesis provides the tumour with amounts of glucose sufficient for the exaggerated glycolysis, and might be the pathway for the high energy expenditure in these patients and, possibly, the sign of cancer cachexia. It has

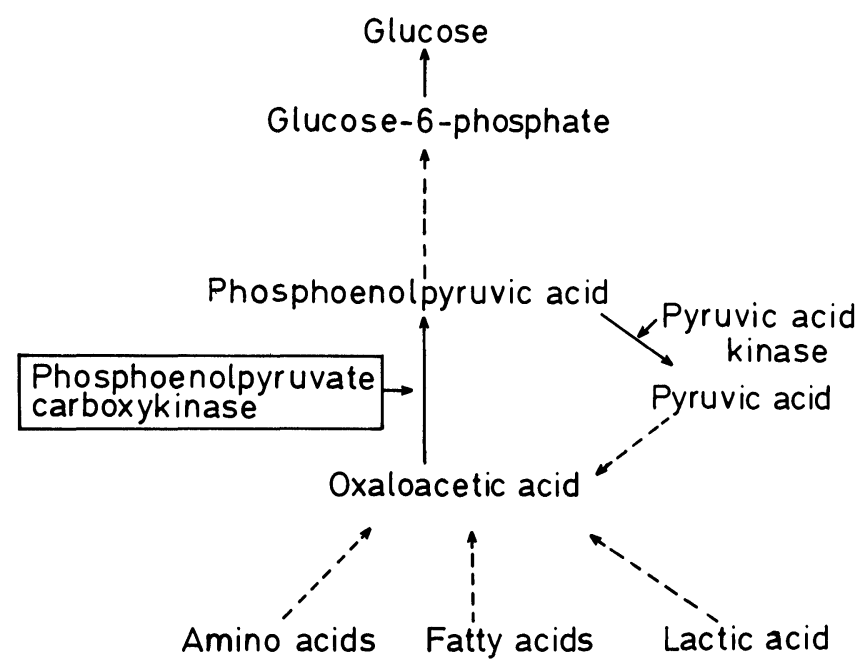

Figure 5 A simplified outline of the metabolic pathways of gluconeogenesis, showing the conversion of amino acids, lipids and carbohydrates to glucose via the tricarboxylic acid cycle intermediates. Illustrated is the enzyme phosphoenolpyruvate carboxykinase, which converts oxaloacetate via pyruvic acid to phosphoenolpyruvic acid, and which is inhibited by hydrazine sulphate. 
therefore been proposed that inhibition of the exaggerated gluconeogenesis would reverse, or at least diminish, cancer cachexia, and also by diminishing the provision of glucose to tumour cells inhibit tumour growth ${ }^{56}$. Theoretically, inhibition of gluconeogenesis could be achieved by inhibition of the enzyme phosphoenolpyruvate carboxykinase, i.e. the enzyme catalyzing the conversion of pyruvic acid to phosphoenolpyruvic acid ${ }^{57}$. By inhibition of this enzyme, gluconeogenesis would be selectively inhibited, without any inhibition of glycolysis, which lacks this enzyme in its pathway, since the conversion of phosphoenolpyruvic acid kinase to pyruvic acid in the glycolytic pathway is catalyzed by pyruvic acid kinase ${ }^{57}$.

Hydrazine sulphate was found to be an agent that specifically inhibits phosphoenolpyruvate carboxykinase; the inhibition was found to be irreversible and readily obtained in vivo as well as in vitro ${ }^{58}$. Hydrazine sulphate belongs to a group of anti-cancer agents, of which the most well known is procarbazine ${ }^{59}$. Hydrazine sulphate has been tested experimentally and found to inhibit growth of a variety of experimental tumours, e.g. Walker 256 carcinosarcoma, Murphy-Sturm lymphosarcoma, B-16 melanoma, and fibrosarcoma and adenocarcinoma of the lung and breast in mice ${ }^{60-63}$. Subsequently, the drug was tested in a group of 84 patients with disseminated cancer and found to improve the patients subjectively in $70 \%$ and objectively in $17 \%$ within three months of treatment ${ }^{64}$. Furthermore, the effects of hydrazine sulphate were evaluated in a group of 233 patients with disseminated tumours during a period of 1-6 months, and the drug was found to reduce tumour growth in $50 \%$ of cases ${ }^{65}$. It also improved cancer cachexia ${ }^{57,66}$. A recent doubleblind trial on 12 malnourished patients with lung cancer also showed that, after just 30 days of treatment, improvement of cancer cachexia was obtained ${ }^{66}$. However, negative results have also been seen: in a study on 25 patients with solid malignant tumours, no subjective or objective improvement was observed during a 4-week treatment period with hydrazine sulphate ${ }^{67}$. It might thus be concluded so far that inhibition of gluconeogenesis by the use of hydrazine sulphate improves metabolic and nutritional indices in patients with cancer cachexia, but it is too early to conclude whether this is combined with reduction in tumour growth and/or improved survival rates $^{67}$. The studies with hydrazine sulphate, however, have illustrated the possibility of interfering with host metabolism, and this, perhaps together with manipulations directed towards tumour metabolism, might turn out a feasible direction for future research.

\section{CONCLUSIONS}

This review has focused on two new approaches for treatment of malignancies with special reference to hepatic malignancies: dearterialization and manipulation of the glucose metabolism. Both these approaches seem promising for the future. However, what is needed now is research undertaken to clarify the exact mechanisms behind the effects obtained by the different treatments, to establish their full consequences for both tumour and host cells, and to identify the clinical use of these treatments and their possible combinations. 


\section{References}

1. Bierman, H.R., Byron, Jr R.L., Kelley, K.H. and Grady, A. (1951) Studies on the blood supply of tumours in man. III. Vascular patterns of the liver by hepatic arteriography in vivo. J. Natl. Cancer Inst., 12, 107-112

2. Breedis, C. and Young, G. (1954) The blood supply of neoplasms in the liver. Am. J. Pathol., 30, 969985

3. Ackerman, N.V., Lien, W.N., Kondi, E.S. and Silverman, N.A. (1969) The blood supply of experimental liver metastases. I. The distribution of hepatic artery and portal vein to "small" and "large" tumours. Surgery, 66, 1067-1072

4. Ackerman, N.V. (1974) The blood supply of experimental liver metastases. IV. Changes in vascularity with increasing tumour growth. Surgery, 75, 589-596

5. Mori, W., Masuda, M. and Miyanaga, T. (1966) Hepatic artery ligation and tumour necrosis in the liver. Surgery, 59, 359-363

6. Nilsson, L.A.V. (1966) Therapeutic hepatic artery ligation in patients with secondary liver tumours. Rev. Surg., 23, 374-376

7. Maton, P.N., Camilleri, M., Griffin, G., Allison, D.J., Hodgson, H.J.F. and Chadwick, V.S. (1983) Role of hepatic arterial embolization in the carcinoid syndrome. Brit. Med. J., 287, 932-935

8. Mårtensson, H., Nobin, A., Bengmark, S., Lunderquist, A., Owman, T. and Sanden, G. (1984) Embolization of the liver in the management of metastatic carcinoid tumours. J. Surg. Oncol., 27, 152-158

9. Bengmark, S. and Rosengren, K. (1970) Angiographic study of the collateral circulation to the liver after ligation of the hepatic artery in man. Am. J. Surg., 119, 620-624

10. Dahl, A.P., Fredlund, P.E., Tylen, U. and Bengmark, S. (1981) Transient hepatic dearterialization followed by regional intraarterial 5-fluorouracil infusion as treatment for liver tumours. Ann. Surg., 192, 82-88

11. Bengmark, S., Ericsson, M., Lunderquist, A., Mårtenson, H., Nobin, A., and Sako, M. (1982) Temporary liver dearterialization in patients with metastatic carcinoid disease. World J. Surg., 6, 4653

12. Persson, B., Jeppsson, B., Ekelund, L. and Bengmark, S. (1984) A new device for temporary occlusion of the hepatic artery. J. Exp. Clin. Cancer Res., 3, 155-160

13. Persson, B., Andersson, L., Jeppsson, B., Ekelund, L., Strand, S.E. and Bengmark, S. (1984) Development of collateral circulation after repeated transient occlusion of the hepatic artery in pigs. Eur. Surg. Res., 16 (suppl 1), 102-103

14. Rockwell, S., Moulder J.E. and Martin, D.F. (1986) Effectiveness and biological effects of techniques used to induce hypoxia in solid tumours. Radiother. Oncol., 5, 311-319

15. Mueller-Klieser, W., Freyer, J.P. and Sutherland, R.M. (1986) Influence of glucose and oxygen supply conditions on the oxygenation of multicellular spheroids. Brit. J. Cancer, 53, 343-353

16. Dave, S., Kallinowski, F. and Vaupel, P. (1985) Blood flow and oxygen supply to human mammary carcinomas transplanted into nude rats. Adv. Exp. Med. Biol, 191, 753-762

17. Denekamp, J. (1984) Vascular endothelium as the vulnerable element in tumours. Acta Radiol. Oncol., 23, 217-225

18. Jennische, E. (1985) Development of early post-ischemic injury in the liver as evaluated by a double staining method combining an intravital due exclusion test and alizarin red S. Acta Pathol. Microbiol. Immunol. Scand., 93, 359-365

19. Frederiks, W.M., Marx, F. and Myagkaya, G.L. (1986) A histochemical study of changes in mitochondrial enzyme activities of rat liver after ischaemia in vitro. Virchows Arch. B, 51, 321-329

20. Okayasu, T., Curtis, M.T. and Farber, J.L. (1985) Structural alterations of the inner mitochondrial membrane in ischemic liver cell injury. Arch. Biochem. Biophys., 236, 638-645

21. Frederiks, W.M., Vogels, I.M. and Fronik, G.M. (1984) Plasma ornithine carbamyl transferase level as an indicator of ischaemic injury of rat liver. Cell Biochem. Funct., 2, 217-220

22. Frederiks, W.M., Myagkaya, G.L., van Veen, H.A. and Vogels, I.M. (1984) Biochemical and ultrastructural changes in rat liver plasma membranes after temporary ischaemia. Virchows Arch. Cell. Pathol., 46, 269-282

23. Dawiskiba, J., Isaksson, B., Jeppsson, B., Hägerstrand, I. and Bengmark, S. (1984) Cytoprotective effect of 16, 16-dimethyl prostaglandin $\left(\mathrm{PGHE}_{2}\right)$ on ischemic splanchnic injuries in the rat. Eur. Surg. Res., 16, 77-83

24. Bengmark, S., Börjesson, B., Fredlund, P.E. and Vang, J.O. (1976) Plasma activities of lysosomal enzymes after hepatic dearterialization in man. Am. J. Surg., 132, 363-367

25. Winkler, K., Keiding, S., Tonnesen, K. and Tygstrup, N. (1986) Effect of short lasting hypoxia on the 
metabolic function of the perfused pig liver. Comparison of ischaemic and hypoxaemic hypoxia. Eur. J. Clin. Invest., 16, 106-112

26. Pritsos, C.A. and Sartorelli, A.C. (1986) Generation of reactive oxygen radicals through bioactivation of mitomycin antibiotics. Cancer Res., 46, 3528-3532

27. Fumarulo, R., Riccardi, S., Restaino, A. and Giordano, D. (1984) Effect of cisplatin on the oxidative metabolism of polymorphonuclear leukocytes in cancer patients. Tumori, 30, 227-229

28. Marubayashi, S., Dahl, K., Ochi, K. and Kawasaki, T. (1986) Role of free radicals in ischemic rat liver cell injury: prevention of damage by alpha-tocopherol administration. Surgery, 99, 184-192

29. Im, M.J., Manson, P.N., Bulkley, G.B. and Hoopes, J.E. (1984) Effects of superoxide dismutase and allopurinol on the survival of acute island skin flaps. Ann. Surg., 201, 357-359

30. Schoenberg, M.H., Muhl, E., Sellin, D., Younes, M., Schildberg, F.W. and Haglund, U. (1984) Posthypotensive generation of superoxide-free radicals - possible role in the pathogenesis of intestinal mucosal damage. Acta Chir. Scand., 150, 301-309

31. Keller, G.A., Barke, R., Harty, J.T., Humphrey, E. and Simmons, R.L. (1985) Decreased hepatic glutathione levels in shock. Arch. Surg., 120, 941-945

32. Nordström, G., Seeman, T. and Hasselgren, P.O. (1985) Beneficial effect of allopurinol in liver ischemia. Surgery, 97, 679-684

33. Jennische, E. and Hansson, H.A. (1984) Disulfiram is protective against postischemic cell death in the liver. Acta Physiol. Scand., 122, 199-201

34. Jennische, E. (1984) Possible influence of glutathione on postischemic liver injury. Acta Pathol. Microbiol. Immunol. Scand., 92, 55-64

35. Bengmark, S., Puntis, M. and Jeppsson, B. (1986) Hepatic dearterialization in cancer: new perspectives. Eur. Surg. Res., 18, 151-158

36. Fornander, J., Bergmark, J., Jagenburg, R. and Hasselgren, P.O. (1985) Evaluation of an in vitro method for the study of hepatic protein synthesis in liver ischemia. Eur. Surg. Res., 17 91-100

37. Fornander, J., Seeman, T. and Hasselgren, P.O. (1985) Changes of protein synthesis in liver tissue following ligation of hepatic artery or portal vein in rats. Eur. Surg. Res., 17, 101-108

38. Sutherland, R., Freyer, J., Mueller-Kliesser, W., Wilson, R., Heacock, C., Sciandra, J. and Sordat, B. (1986) Cellular growth and metabolic adaptations to nutrient stress environments in tumor microregions. Int. J. Radiat. Oncol. Biol. Phys., 12, 611-615

39. Denekamp, J., Hill, S. and Hobson, B. (1983) Vascular occlussion and tumour cell death. Eur. J. Cancer Clin. Oncol., 19, 271-279

40. Mack, P. (in press) Retarding liver cancer growth in the rat by transient repeated hepatic dearterialization. J. Surg. Res.

41. Warbug, O. (1931)The Metabolism of Tumors. London: Constable

42. Bustamente, E., Morris, H.P. and Pedersen, P.L. (1981) Energy metabolism of tumour cells. Requirement for a form of hexokinase with a propensity for mitochondrial binding. J. Biol. Chem., 256, 8699-8704

43. Holroyde, C.P., Gabuzda, T.G., Putnam R.C., Paul, P. and Reichard, G.A. (1975) Altered glucose metabolism in metastatic cancer. Cancer Res., 35, 3710-3714

44. Lundholm, K., Edström, S., Karlberg, I., Ekman, L. and Scherstén, T. (1982) Glucose turnover, gluconeogenesis from glycerol, and estimation of net glucose cycling in cancer patients. Cancer, $\mathbf{5 0}$, $1142-1150$

45. Wick, N.R., Drury, R., Naxada, H.L. and Wolff, J.B. (1957) Localization of the primary metabolic block produced by 2-DG. J. Biol. Chem., 244, 963-969

46. Cramer, F.B. and Woodward, G.E. (1979) 2-Deoxy-D-glucose as an antagonist of glucose in yeast fermentation. J. Franklin Inst., 253, 354-360

47. Chen, M. and Whistler, R.L. (1957) Action of 5-thio-D-glucose and its 1-phosphate with hexokinase and phosphoglucomutase. Arch. Biochem. Biophys., 169, 392-396

48. Laszlo, J., Landau, B., Wight, K. and Burk, D. (1958) The effect of glucose analogues on the metabolism of human leukemic cells. J. Natl. Cancer Inst., 21, 474-483

49. Jain, V.K., Kajia, V.K., Sharma, R., Maharajan, V. and Menon, M. (1985) Effects of 2-deoxy-Dglucose on glycolysis, proliferation kinetics and radiation response of human cancer cells. Int. $J$. Radiat. Oncol. Biol. Phys., 11, 943-950

50. Laszlo, J., Humphreys, S.R. and Goldin, A. (1960) Effects of glucose analogues (2-deoxy-D-glucose, 2-deoxy-D-galactose) on experimental tumours. J. Natl. Cancer Inst., 24, 267-279

51. Kern, K.A. and Norton, J.A. (1987) Inhibition of established rat fibrosarcom growth by the glucose antagonist 2-deoxy-D-glucose. Surgery, 102, 380-385

52. Tannock, I.F., Guttman, P. and Rauth, A.M. (1983) Failure of 2-deoxy-D-glucose and 5-thio-Dglucose to kill hypoxic cells of two murine tumours. Cancer Res., 43, 980-983 
53. Taborsky, Jr G.J., Halter, J.B. and Porte, Jr D. (1982) Morphine suppresses plasma catecholamine responses to laparotomy but not to 2-deoxy-glucose. Am. J. Physiol., 242, E317-E322

54. Karlsson, S., Bood, M. and Ahrén, B. (1987) The mechanism of 2-deoxy-glucose-induced insulin secretion in the mouse. J. Auton. Pharmacol., 7, 135-144

55. Gold, J. (1968) Proposed treatment of cancer by inhibition of gluconeogenesis. Oncology, 22, 185207

56. Chlebowski, R.T., Heber, D., Richardson, B. and Block, J.B. (1984) Influence of hydrazine sulfate on abnormal carbohydrate metabolism in cancer patients with weight loss. Cancer Res., 44, 857-861

57. Gold, J. (1987) Hydrazine sulfate: a current perspective. Nutrition Canc., 9, 59-66

58. Ray, P.D., Hanson, R.L. and Lardy, H.A. (1970) Inhibition by hydrazine of gluconeogenesis in the rat. J. Biol. Chem., 245, 690-696

59. Tweedie, D.J., Erikson, J.M. and Prough, R.A. (1987) Metabolism of hydrazine anticancer agents. Pharmac. Ther., 34, 111-127

60. Gold, J. (1971) Inhibition of Walker 256 intramuscular carcinoma in rats by administration of hydrazine sulfate. Oncology, 25, 66-71

61. Gold, J. (1973) Inhibition by hydrazine sulfate and various hydrazides, of in vivo growth of Walker 256 intramuscular carcinoma, B-16 melanoma, Murphy-Sturm lymphosarcoma and L-1210 solid leukemia. Oncology, 27, 69-80

62. Seits, J.F., Gershanovich, M.L. Filov, V.A., Danova, L.A., Kondratyev, V.B., Malyugina, L.L., Muller, N.R., Polikna, R.I., Rudakov, V.G. and Stukov, A.N. (1975) Experimental and clinical data on the antitumor action of hydrazine sulfate. Vopr. Onkol., 21, 45-52

63. Bhide, S.V. (1978) Anticancer properties of isoniazid and hydrazine sulfate. Indian J. Exp. Biol., 16, 926-928

64. Gold, J. (1975) Use of hydrazine sulfate in terminal and preterminal cancer patients: results of investigational new drug (IND) study in 84 evaluable patients. Oncology, 32, 1-10

65. Gershanovich, M.L., Danova, L.A., Ivin, B.A. and Filov, V.A. (1981) Results of clinical study of antitumor action of hydrazine sulfate. Nutrition Canc., 3, 7-12

66. Tayek, J.A., Heber, D. and Chlebowski, R.T. (1987) Effect of hydrazine sulphate on whole-body protein breakdown measured by ${ }^{14} \mathrm{C}$-lysine metabolism in lung cancer patients. Lancet, ii, 241-244

67. Chlebowski, R.T. and Heber, D. (1986) Metabolic abnormalities in cancer patients: carbohydrate metabolism, Surg. Clin. N. Amer., 66, 957-968 


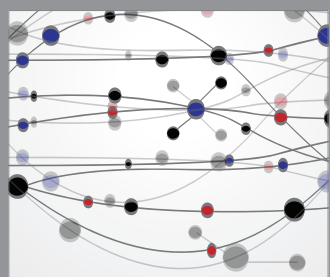

The Scientific World Journal
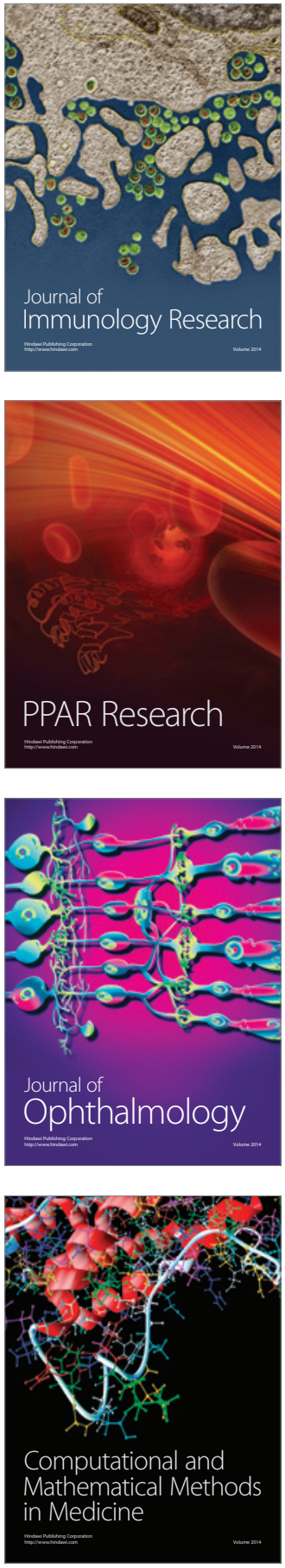

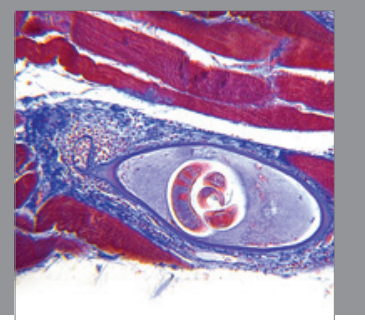

Gastroenterology

Research and Practice
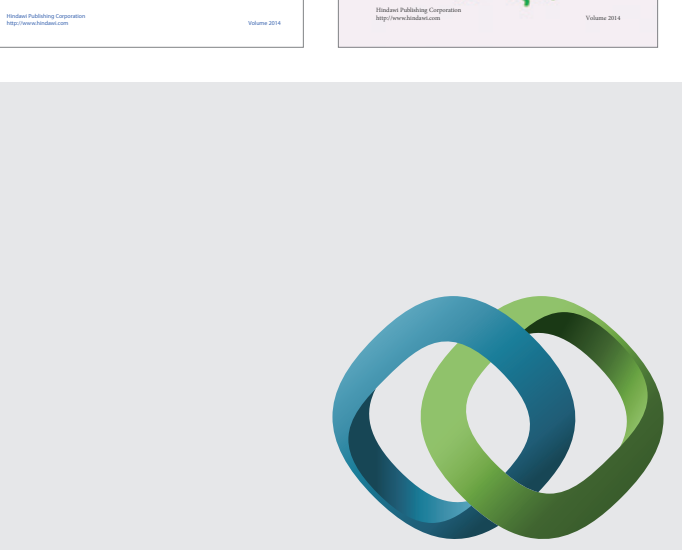

\section{Hindawi}

Submit your manuscripts at

http://www.hindawi.com
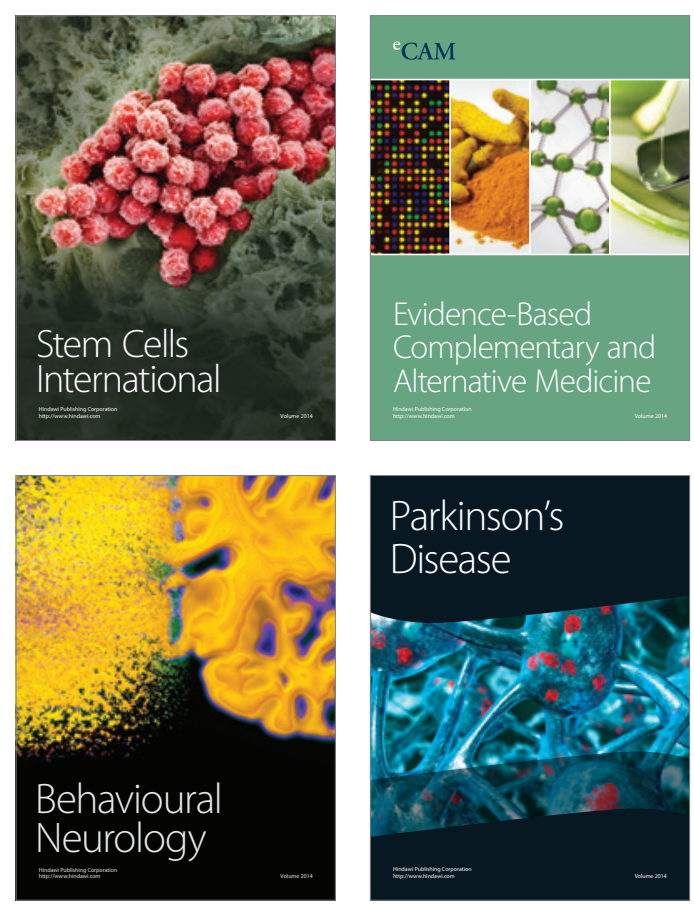

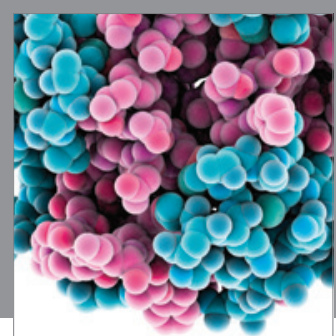

Journal of
Diabetes Research

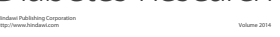

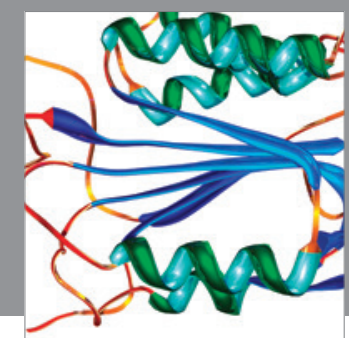

Disease Markers
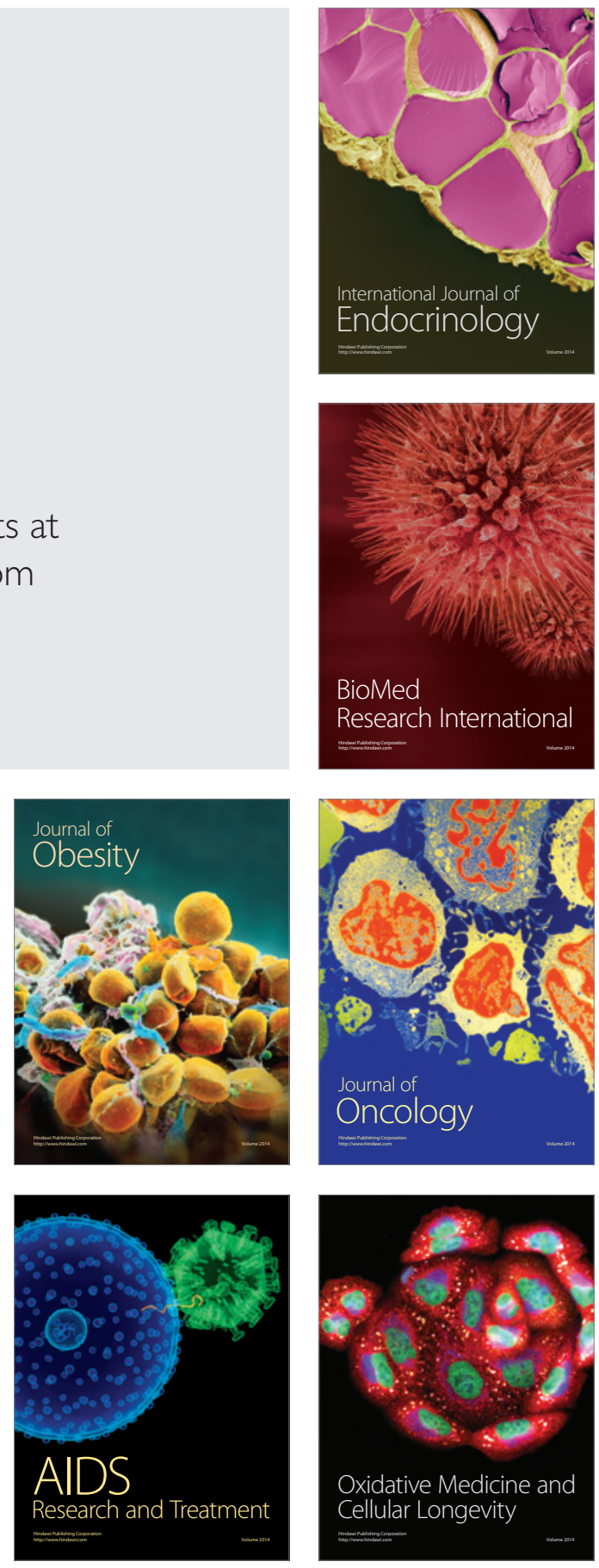